\title{
BIORTHOGONAL INTERPOLATORY MULTISCALING FUNCTIONS AND CORRESPONDING MULTIWAVELETS
}

\author{
YANG SHOUZHI ${ }^{1}$
}

(Received 2 May, 2004; revised 30 June, 2006)

\begin{abstract}
A method for constructing a pair of biorthogonal interpolatory multiscaling functions is given and an explicit formula for constructing the corresponding biorthogonal multiwavelets is obtained. A multiwavelet sampling theorem is also established. In addition, we improve the stability of the biorthogonal interpolatory multiwavelet frame by the linear combination of a pair of biorthogonal interpolatory multiwavelets. Finally, we give an example illustrating how to use our method to construct biorthogonal interpolatory multiscaling functions and corresponding multiwavelets.
\end{abstract}

2000 Mathematics subject classification: primary 42C15; secondary 94A12.

Keywords and phrases: biorthogonal, interpolatory, multiscaling functions, multiwavelets, frame.

\section{Introduction}

In recent years, multiscaling functions and multiwavelets have been studied extensively (see $[1,2,4,5,7,8]$ ). Recently, biorthogonal multiwavelets have also been the subject of considerable interest. In $[6,10-12]$, methods for constructing compactly supported biorthogonal multiwavelets were given.

Sampling theorems play a fundamental role in digital signal processing. The classical Shannon sampling theorem declares that bandlimited signals can be exactly represented by their uniform samples as long as the sampling rate is not less than the Nyquist rate. This theorem has been widely used in many fields, and generalized into many different forms. For example, some wavelet sampling theorems have been established within the last decade $[14,18]$. In [18], Zhou investigated interpolatory orthogonal multiscaling functions and multiwavelets, and established a multiwavelets sampling theorem. In this paper, we study how to construct biorthogonal interpolatory multiscaling functions and corresponding multiwavelets.

\footnotetext{
'Dept. of Maths., Shantou University, Shantou 515063, P.R. China; email: szyang@stu.edu.cn.

(C) Australian Mathematical Society 2007, Serial-fee code 1446-1811/07
} 
The paper is organized as follows: In Section 2, we briefly recall the concept of multiresolution analysis. In Section 3, we introduce a procedure for constructing biorthogonal interpolatory multiscaling functions, and establish a multiwavelets sampling theorem. In Section 4, we give a method for constructing biorthogonal interpolatory multiwavelets. In Section 5, we improve the stability of the biorthogonal interpolatory multiwavelets frame by the linear combination of a pair of biorthogonal interpolatory multiwavelets. In Section 6, we give an example illustrating how to use our method to construct a pair of biorthogonal interpolatory multiscaling functions and corresponding multiwavelets.

\section{Multiresolution Analysis}

Let $\Phi(x)=\left(\phi_{1}, \phi_{2}\right)^{T}, \phi_{1}, \phi_{2} \in L^{2}(R)$, satisfy the two-scale matrix equation

$$
\Phi(x)=\sum_{k \in Z} P_{k} \Phi(2 x-k),
$$

where $\left\{P_{k}\right\}$ is a finitely supported sequence of $2 \times 2$ matrices called the two-scale matrix sequence of $\boldsymbol{\Phi}$. We call $\Phi(x)$ a multiscaling function with multiplicity two.

By taking the Fourier transform of both sides of (2.1), we have

$$
\hat{\boldsymbol{\Phi}}(w)=P(z) \hat{\boldsymbol{\Phi}}\left(\frac{w}{2}\right), \quad z=e^{-i w / 2},
$$

where $P(z)=(1 / 2) \sum_{k \in Z} P_{k} z^{k}$ is called the two-scale matrix symbol of $\left\{P_{k}\right\}$ of $\Phi$.

Define a subspace $V_{j} \subset L^{2}(R)$ by

$$
V_{j}=\operatorname{clos}_{L^{2}(R)}\left\langle\phi_{\ell: j, k}(x): \ell=1,2 ; k \in Z\right\rangle, \quad j \in Z .
$$

Henceforth, for $f_{\ell}(x) \in L^{2}$, we will use the notation $f_{\ell: j, k}(x)=2^{j / 2} f_{\ell}\left(2^{j} x-k\right)$. Let $W_{j}, j \in Z$, denote the complementary subspace of $V_{j}$ in $V_{j+1}$, and let the vectorvalued function $\Psi(x)=\left(\psi_{1}(x), \psi_{2}(x)\right)^{T}, \psi_{\ell}(x) \in L^{2}, \ell=1,2$ constitute a Riesz basis for $W_{j}$, that is,

$$
W_{j}=\operatorname{clos}_{L^{2}(R)}\left\langle\psi_{\ell: j, k}(x): \ell=1,2 ; k \in Z\right\rangle, \quad j \in Z .
$$

It is clear that $\psi_{1}(x)$ and $\psi_{2}(x)$ are in $W_{0} \subset V_{1}$. Hence there exists a sequence of matrices $\left\{Q_{k}\right\}_{k \in Z}$ such that

$$
\Psi(x)=\sum_{k \in Z} Q_{k} \Phi(2 x-k)
$$

By the two-scale relation (2.2) of $\boldsymbol{\Psi}(x)$, we have $\hat{\boldsymbol{\Psi}}(w)=Q(z) \hat{\boldsymbol{\Phi}}(w / 2)$, where $Q(z)=(1 / 2) \sum_{k \in Z} Q_{k} z^{k}$. 
We call $\Phi(x)=\left(\phi_{1}(x), \phi_{2}(x)\right)^{T}$ and $\tilde{\boldsymbol{\Phi}}(x)=\left(\tilde{\phi}_{1}(x), \tilde{\phi}_{2}(x)\right)^{T}$ a pair of biorthogonal multiscaling functions, if $\langle\Phi(\cdot), \tilde{\Phi}(\cdot-n)\rangle=\delta_{0, n} I_{2}, n \in Z$. The functions $\boldsymbol{\Psi}(x)=\left(\psi_{1}(x), \psi_{2}(x)\right)^{T}$ and $\tilde{\boldsymbol{\Psi}}(x)=\left(\tilde{\psi}_{1}(x), \tilde{\psi}_{2}(x)\right)^{T}$ will be said to be a pair of biorthogonal multiwavelets associated with $\Phi$ and $\tilde{\boldsymbol{\Phi}}$, if $\boldsymbol{\Phi}, \tilde{\boldsymbol{\Phi}}$ and $\Psi, \tilde{\Psi}$ satisfy

$$
\langle\boldsymbol{\Phi}(\cdot), \tilde{\boldsymbol{\Psi}}(\cdot-n)\rangle=\langle\boldsymbol{\Psi}(\cdot), \tilde{\boldsymbol{\Phi}}(\cdot-n)\rangle=O \quad \text { and }\langle\boldsymbol{\Psi}(\cdot), \tilde{\boldsymbol{\Psi}}(\cdot-n)\rangle=\delta_{0, n} I_{2},
$$

for $n \in Z$, where $O$ and $I_{2}$ denote the zero and identity matrices, respectively.

For simplicity, let $\tilde{P}(z)$ and $\tilde{Q}(z)$ be the two-scale symbol matrices of $\tilde{\boldsymbol{\Phi}}$ and $\tilde{\Psi}$, respectively.

\section{Biorthogonal Interpolatory Multiscaling Functions}

A multiscaling function $\Phi(x)$ satisfying (2.1) is called interpolatory if $\phi_{1}(x)$ and $\phi_{2}(x)$ are continuous, compactly supported and satisfy for $k \in Z, \ell=0,1$,

$$
\phi_{j}\left(k+\frac{\ell}{2}\right)=\delta_{k, 0} \delta_{j, \ell+1}, \quad j=1,2 .
$$

The interpolatory condition (3.1) means that $\phi_{1}(x)$ is cardinal at integers and vanishes at half integers; $\phi_{2}(x)$ is cardinal at half integers and vanishes at integers.

Let $\mathbf{e}_{1}=(1,0)^{T}$ and $\mathbf{e}_{2}=(0,1)^{T}$. Then the interpolatory condition (3.1) is equivalent to the equations

$$
\boldsymbol{\Phi}(k)=\delta_{k, 0} \mathbf{e}_{1} \quad \text { and } \quad \boldsymbol{\Phi}\left(k+\frac{1}{2}\right)=\delta_{k, 0} \mathbf{e}_{2} .
$$

By (2.1) and (3.2), we obtain

$$
\boldsymbol{\Phi}(k)=\delta_{k, 0} \mathbf{e}_{1}=P_{2 k} \mathbf{e}_{1} \text { and } \boldsymbol{\Phi}\left(k+\frac{1}{2}\right)=\delta_{k, 0} \mathbf{e}_{2}=P_{2 k+1} \mathbf{e}_{1} .
$$

Equation (3.3) means that the first column of matrices $P_{k}$ can be obtained. Hence

$$
P(z) \mathbf{e}_{1}=\frac{1}{2}\left[\sum_{k} P_{2 k} \mathbf{e}_{1} z^{2 k}+\sum_{k} P_{2 k+1} \mathbf{e}_{1} z^{2 k+1}\right]=\frac{1}{2}\left[\begin{array}{l}
1 \\
z
\end{array}\right] .
$$

By (3.4), we know that if a multiscaling function $\Phi(x)$ possesses the interpolatory property, then the first column of its two-scale matrix symbol $P(z)$ must have the form $(1 / 2)[1, z]^{T}$.

Next, we suppose that a pair of biorthogonal multiscaling functions $\Phi(x)$ and $\tilde{\Phi}(x)$ are both interpolatory. Then the two-scale matrix symbols have the respective forms

$$
P(z)=\frac{1}{2}\left[\begin{array}{cc}
1 & H(z) \\
z & G(z)
\end{array}\right] \text { and } \tilde{P}(z)=\frac{1}{2}\left[\begin{array}{cc}
1 & \tilde{H}(z) \\
z & \tilde{G}(z)
\end{array}\right]
$$


where $H(z), G(z), \tilde{H}(z)$ and $\tilde{G}(z)$ are all Laurent polynomials.

Let $\boldsymbol{\Phi}(x)$ and $\tilde{\boldsymbol{\Phi}}(x)$ be a pair of biorthogonal multiscaling functions, with twoscale matrix symbols $P(z)$ and $\tilde{P}(z)$, respectively. Then $P(z)$ and $\tilde{P}(z)$ satisfy the following identity $[12]$ :

$$
P(z) \tilde{P}(z)^{*}+P(-z) \tilde{P}(-z)^{*}=I_{2}, \quad|z|=1 .
$$

Here and throughout, the asterisk denotes complex conjugation of the transpose.

In connection with Equation (3.5), Equation (3.6) is equivalent to $H(z), G(z), \tilde{H}(z)$ and $\tilde{G}(z)$ satisfying the following four equations:

$$
\begin{aligned}
H(z) \tilde{H}(z)^{*}+H(-z) \tilde{H}(-z)^{*} & =2, \\
G(z) \tilde{G}(z)^{*}+G(-z) \tilde{G}(-z)^{*} & =2, \\
H(z) \tilde{G}(z)^{*}+H(-z) \tilde{G}(-z)^{*} & =0, \\
G(z) \tilde{H}(z)^{*}+G(-z) \tilde{H}(-z)^{*} & =0 .
\end{aligned}
$$

To construct $P(z)$ and $\tilde{P}(z)$, that is, $H(z), G(z), \tilde{H}(z)$ and $\tilde{G}(z)$, we need the following theorem.

THEOREM 3.1. Let $\boldsymbol{\Phi}(x)$ and $\tilde{\boldsymbol{\Phi}}(x)$ be a pair of biorthogonal multiscaling functions, with two-scale matrix symbols $P(z)$ and $\tilde{P}(z)$, respectively. Then condition (3.6) holds if and only if Equation (3.7) holds and

$$
G(z)=g z^{2 p+1} \tilde{H}(-z)^{*}, \quad \tilde{G}(z)=\frac{1}{\bar{g}} z^{2 p+1} H(-z)^{*},
$$

where $g$ is nonzero constant and $p$ is in $Z$.

Further, $\Phi(x)$ and $\tilde{\boldsymbol{\Phi}}(x)$ both satisfy the following equations:

$$
\boldsymbol{\Phi}(k)=\tilde{\boldsymbol{\Phi}}(k)=\delta_{k, 0} \mathbf{e}_{1}, \quad \boldsymbol{\Phi}\left(k+\frac{1}{2}\right)=\tilde{\boldsymbol{\Phi}}\left(k+\frac{1}{2}\right)=\delta_{k, 0} \mathbf{e}_{2}, \quad k \in Z
$$

PROOF. Note that if a Laurent polynomial vanishes on the unit circle, then it is identically zero on $C \backslash\{0\}$. Let $P(z)$ and $\tilde{P}(z)$ be the two-scale matrix symbols defined in Equation (3.5). Suppose that condition (3.6) holds. Hence, $\forall z \in C \backslash\{0\}$

$$
\begin{aligned}
P(z) & \tilde{P}(z)^{*}+P(-z) \tilde{P}(-z)^{*} \\
\quad= & \frac{1}{4}\left[\begin{array}{cc}
2+H(z) \tilde{H}(z)^{*}+H(-z) \tilde{H}(-z)^{*} & H(z) \tilde{G}(z)^{*}+H(-z) \tilde{G}(-z)^{*} \\
G(z) \tilde{H}(z)^{*}+G(-z) \tilde{H}(-z)^{*} & 2+G(z) \tilde{G}(z)^{*}+G(-z) \tilde{G}(-z)^{*}
\end{array}\right] \\
= & I_{2} .
\end{aligned}
$$


Comparison of the entries of this equation provides (3.7)-(3.10). It is clear that $H(z)$ and $H(-z)$ have no common zero in $C \backslash\{0\}$. Since $H(z) \tilde{G}(z)^{*}+H(-z) \tilde{G}(-z)^{*}=0$, $|z|=1$, there exists a Laurent polynomial $\tilde{g}(z)$ such that

$$
\tilde{G}(-z)^{*}=\tilde{g}(z) H(z), \quad|z|=1 .
$$

By Equation (3.7), $\tilde{H}(z)$ and $\tilde{H}(-z)$ have also no common zero in $C \backslash\{0\}$. Similarly, there exists a Laurent polynomial $\tilde{h}(z)$ such that

$$
H(-z)=\tilde{h}(z) \tilde{G}(z)^{*}, \quad|z|=1 .
$$

Considering Equations (3.8), (3.12) and (3.13), we have

$$
H(z) \tilde{G}(z)^{*}[1+\tilde{g}(z) \tilde{h}(z)]=0, \quad|z|=1 .
$$

Since neither $H(z)$ nor $\tilde{G}(z)^{*}$ is identically zero, $\tilde{g}(z) \tilde{h}(z)=-1, \forall z \in C \backslash\{0\}$. This means that $\tilde{g}(z)$ and $\tilde{h}(z)$ have no zero in $C \backslash\{0\}$. Therefore, let $\tilde{g}(z)=\tilde{g} z^{\tilde{s}}$, where $\tilde{g}$ is some nonzero constant and $\tilde{s} \in Z$. Then $\tilde{h}(z)=-(1 / \tilde{g}) z^{-\tilde{s}}$.

Similarly, there exist two Laurent polynomials $g(z)$ and $h(z)$ such that

$$
G(-z)^{*}=g(z) \tilde{H}(z), \quad \tilde{H}(-z)=h(z) G(z)^{*}, \quad|z|=1,
$$

where $g(z)=g z^{s}$ and $h(z)=-(1 / g) z^{s}$.

Hence we have

$$
\begin{aligned}
G(z) \tilde{G}(z)^{*}+G(-z) \tilde{G}(-z)^{*} & =g \tilde{g} z^{s-\tilde{s}}\left[H(z) \tilde{H}(z)^{*}+H(-z) \tilde{H}(-z)^{*}\right] \\
& =2 g \tilde{g} z^{s-\tilde{s}}=2 .
\end{aligned}
$$

Therefore $g \tilde{g}=1$ and $s=\tilde{s}$.

By Equation (3.13), we have

$$
H(-z)=\tilde{h}(z) \tilde{G}(z)^{*}=-\frac{1}{\tilde{g}} z^{-\tilde{s}} \tilde{g}(-z)^{s} H(-z)=(-1)^{s+1} H(-z) .
$$

However, $H(-z)$ is not identically zero. Hence $s$ is odd. Therefore

$$
G(z)=g z^{2 p+1} \tilde{H}(-z)^{*} \quad \text { and } \quad \tilde{G}(z)=\frac{1}{g} z^{2 p+1} H(-z)^{*}
$$

for some nonzero constant $g$ and $p \in Z$. This proves condition (3.11) of Theorem 3.1.

Conversely, if (3.7) and (3.11) are true, then condition (3.6) can be easily obtained.

Next, we prove the second statement of Theorem 3.1. Suppose that $\Phi(x)$ satisfies (2.1). Noticing that $P(z)$ defined in (3.5) is the symbol of the sequence $\left\{P_{k}\right\}$ associated with $\Phi(x)$ and applying (2.1), we have

$$
\boldsymbol{\Phi}(k)=\sum_{\ell \in Z} P_{\ell} \boldsymbol{\Phi}(2 k-\ell)=P_{2 k} \mathbf{e}_{1}=\delta_{k, 0} \mathbf{e}_{1}, \quad k \in Z .
$$


Similarly,

$$
\boldsymbol{\Phi}\left(k+\frac{1}{2}\right)=\sum_{\ell \in Z} P_{\ell} \boldsymbol{\Phi}(2 k+1-\ell)=P_{2 k+1} \mathbf{e}_{1}=\delta_{k, 0} \mathbf{e}_{2}, \quad k \in Z .
$$

In the same way,

$$
\begin{gathered}
\tilde{\boldsymbol{\Phi}}(k)=\sum_{\ell \in Z} \tilde{P}_{\ell} \tilde{\boldsymbol{\Phi}}(2 k-\ell)=\tilde{P}_{2 k} \mathbf{e}_{1}=\delta_{k, 0} \mathbf{e}_{1}, \quad k \in Z \\
\tilde{\boldsymbol{\Phi}}\left(k+\frac{1}{2}\right)=\sum_{\ell \in Z} \tilde{P}_{\ell} \tilde{\boldsymbol{\Phi}}(2 k+1-\ell)=\tilde{P}_{2 k+1} \mathbf{e}_{1}=\delta_{k, 0} \mathbf{e}_{2}, \quad k \in Z
\end{gathered}
$$

This means that $\boldsymbol{\Phi}(x)$ and $\tilde{\Phi}(x)$ are interpolatory. This completes the proof.

Next we characterize interpolatory multiscaling functions. Our characterization is based on the joint spectral radius which was defined by Rota and Strang [9] and which was introduced into the investigation of wavelets by Daubechies and Lagarias [3].

Let $\mathscr{A}$ be a set of $N \times N$ matrices. With an arbitrary matrix norm $\|\cdot\|$, the joint spectral radius of $\mathscr{A}$ is defined to be

$$
\rho_{\infty}(\mathscr{A})=\lim _{n \rightarrow \infty} \max \left\{\left\|A_{1} A_{2} \cdots A_{n}\right\|^{1 / n}: A_{1}, A_{2}, \ldots, A_{n} \in \mathscr{A}\right\}
$$

For the computation of the joint spectral radius, see $[13,16]$.

Our characterization involves the joint spectral radius of two matrices $\mathscr{A}$ restricted to certain common invariant subspaces. For $v \in C^{N}$, denote $V(v)$ as the minimal subspace containing $v$, which is invariant under each matrix in $\mathscr{A}$. Then $\rho_{\infty}\left(\left.\mathscr{A}\right|_{V(v)}\right)$ denotes the joint spectral radius of the restrictions of matrices in $\mathscr{A}$ to $V(v)$.

In our case, the elements in $\mathscr{A}$ are two matrices. Suppose that $\left\{P_{k}\right\}$ is a sequence of $2 \times 2$ matrices supported on $\left[N_{1}, N_{2}\right]$ with $N_{1}<N_{2}$ : two integers. Then two matrices are defined by

$$
A_{j}=\left[P_{2 k-\ell+j}\right]_{k, \ell=N_{1}}^{N_{2}-1}, \quad j=0,1
$$

Given a two-scale matrix sequence, the existence of compactly supported distributional solutions of the two-scale matrix equation (2.1) can be checked by the criteria in [15]. Considering a continuous solution, its function values at integers $v(j)=\Phi(j)$ satisfy

$$
\sum_{k \in Z} P_{2 j-k} v(k)=v(j), \quad \forall j \in Z
$$

In [17], it was shown that for a two-scale matrix sequence $P_{k}$ supported on $\left[N_{1}, N_{2}\right]$ and a finitely supported sequence of 2 -vectors satisfying (3.14), there exists a vector 
$\boldsymbol{\Phi}(x)=\left[\phi_{1}(x), \phi_{2}(x)\right]^{T}$ of compactly supported continuous functions on $R$ such that (2.1) holds, and $\boldsymbol{\Phi}(k)=v(k)$ if and only if $v$ is supported in $\left[N_{1}+1, N_{2}-1\right]$, and

$$
\rho_{\infty}\left(\left\{\left.A_{0}\right|_{v(\nabla v)}, A_{1} \mid v(\nabla v)\right\}\right)<1
$$

Here $\nabla v=v-v(\cdot+1)$. In this case, $\boldsymbol{\Phi}(x)$ is supported in $\left[N_{1}, N_{2}\right]$.

In [18], applying the above conclusion with $v(j)=\delta_{j, 0} \mathbf{e}_{1}$, a necessary and sufficient condition for the two-scale matrix equation (2.1) to have a continuous compactly supported solution $\boldsymbol{\Phi}(x)$ satisfying interpolatory condition (3.1) was established.

THEOREM 3.2. Suppose that $P(z), \widetilde{P}(z)$ defined in (3.5) are 2 sequences of $2 \times 2$ matrices supported by $\left[N_{1}, N_{2}\right],\left[\widetilde{N}_{1}, \tilde{N}_{2}\right]$, respectively. Then $P(z), \widetilde{P}(z)$ can generate a pair of biorthogonal interpolatory multiscaling functions if and only if the following conditions hold:

(a) $N_{1}<0<N_{2}, \widetilde{N}_{1}<0<\tilde{N}_{2}$;

(b) $\rho_{\infty}\left(\left\{\left.A_{0}\right|_{v(\nabla \delta)},\left.A_{1}\right|_{v(\nabla \delta)}\right\}\right)<1, \rho_{\infty}\left(\left\{\left.\tilde{A}_{0}\right|_{v(\nabla \delta)},\left.\tilde{A}_{1}\right|_{v(\nabla \delta)}\right\}\right)<1$, where $\delta$ is the delta sequence;

(c) $P(z), \widetilde{P}(z)$ defined in (3.5) satisfy (3.7) and (3.11).

PROOF. According to Theorem 5.1 of [18], $P(z)$ and $\tilde{P}(z)$ can generate two interpolatory multiscaling functions $\Phi(x)$ and $\tilde{\boldsymbol{\Phi}}(x)$ if and only if conditions (a) and (b) hold. Again by Theorem $3.1, \boldsymbol{\Phi}(x)$ and $\tilde{\boldsymbol{\Phi}}(x)$ constitute a pair of biorthogonal multiscaling functions if and only if condition (c) holds.

Sampling theorems play a fundamental role in digital signal processing. Next, we give a multiwavelet sampling theorem based on a pair of biorthogonal interpolatory multiscaling functions.

THEOREM 3.3. Let $\boldsymbol{\Phi}(x)=\left(\phi_{1}(x), \phi_{2}(x)\right)^{T}$ and $\tilde{\boldsymbol{\Phi}}(x)=\left(\tilde{\phi}_{1}(x), \tilde{\phi}_{2}(x)\right)^{T}$ be a pair of biorthogonal multiscaling functions. If the two functions have the same interpolatory property, and satisfy the interpolatory condition (3.1), then for any continuous signal $\mathbf{f}(x) \in V_{N}$, we have

$$
\mathbf{f}(x)=\sum_{\ell \in Z} 2^{-N / 2}\left[\mathbf{f}\left(\frac{\ell}{2^{N}}\right), \mathbf{f}\left(\frac{\ell}{2^{N}}+\frac{1}{2^{N+1}}\right)\right] \boldsymbol{\Phi}_{N, \ell}(x)
$$

Similarly, for any any continuous signal $\mathbf{g}(x) \in \widetilde{V}_{N}$, we have

$$
\mathbf{g}(x)=\sum_{\ell \in Z} 2^{-N / 2}\left[\mathbf{g}\left(\frac{\ell}{2^{N}}\right), \mathbf{g}\left(\frac{\ell}{2^{N}}+\frac{1}{2^{N+1}}\right)\right] \tilde{\boldsymbol{\Phi}}_{N, \ell}(x)
$$


PROOF. Let $V_{N}$ be the closed subspace spanned by

$$
\left\{\Phi_{N, \ell}(x)=2^{N / 2} \Phi\left(2^{N} x-\ell\right), \quad \ell \in Z\right\} .
$$

If a continuous signal $f(x) \in V_{N}$, then it has the following decomposition:

$$
\mathbf{f}(x)=\sum_{\ell \in Z} \mathbf{C}_{N, \ell}^{T} \boldsymbol{\Phi}_{N, \ell}(x),
$$

with each $\mathbf{C}_{N, \ell}^{T}$ being a 2-vector. According to the interpolatory property (3.1) or (3.2), the coefficients $\mathbf{C}_{N, \ell}^{T}$ can be exactly given by the samples of the signal

$$
\mathbf{C}_{N, \ell}^{T}=2^{-N / 2}\left[\mathbf{f}\left(\frac{\ell}{2^{N}}\right), \mathbf{f}\left(\frac{\ell}{2^{N}}+\frac{1}{2^{N+1}}\right)\right] .
$$

This means (3.15) holds. Similarly, we can prove that (3.16) also holds.

\section{Biorthogonal Interpolatory Multiwavelets}

In the previous section, we gave a method for constructing biorthogonal multiscaling functions with multiplicity 2 using the matrix symbols $P(z)$ and $\tilde{P}(z)$. In this section, we construct the corresponding biorthogonal interpolatory multiwavelets.

Let $\Phi(x)$ and $\tilde{\boldsymbol{\Phi}}(x)$ be a pair of biorthogonal multiscaling functions and let $P(z)$ and $\tilde{P}(z)$ be two-scale matrix symbols. Suppose $\Psi(x)$ and $\tilde{\Psi}(x)$ are a pair of biorthogonal multiwavelets associated with $\boldsymbol{\Phi}(x)$ and $\tilde{\boldsymbol{\Phi}}(x)$, respectively, and $Q(z)$ and $\tilde{Q}(z)$ are the corresponding two-scale matrix symbols. Then

$$
\left\{\begin{array}{l}
P(z) \tilde{Q}(z)^{*}+P(-z) \tilde{Q}(-z)^{*}=O \\
\tilde{P}(z) Q(z)^{*}+\tilde{P}(-z) Q(-z)^{*}=O \\
Q(z) \tilde{Q}(z)^{*}+Q(-z) \tilde{Q}(-z)^{*}=I_{2}
\end{array}\right.
$$

To construct biorthogonal interpolatory multiwavelets associated with $\boldsymbol{\Phi}(x)$ and $\tilde{\boldsymbol{\Phi}}(x)$, define two matrices $Q(z)$ and $\tilde{Q}(z)$ respectively by

$$
\begin{aligned}
& Q(z)=\frac{1}{2}\left[\begin{array}{cc}
1 & -H(z) \\
z & -g z^{2 p+1} \tilde{H}(-z)^{*}
\end{array}\right] \text { and } \\
& \tilde{Q}(z)=\frac{1}{2}\left[\begin{array}{cc}
1 & -\tilde{H}(z) \\
z & -\frac{1}{\bar{g}} z^{2 p+1} H(-z)^{*}
\end{array}\right] .
\end{aligned}
$$


THEOREM 4.1. Let $P(z)$ and $\tilde{P}(z)$ defined in (3.5) be 2 two-scale matrix symbols and let $G(z)$ and $\tilde{G}(z)$ be given by (3.11). Also let $H(z)$ and $\tilde{H}(z)$ satisfy (3.7). Let $\Phi(x)$ and $\tilde{\Phi}(x)$ be a pair of biorthogonal multiscaling functions with two-scale matrix symbols $P(z)$ and $\tilde{P}(z)$, respectively. Then the symbols $Q(z)$ and $\tilde{Q}(z)$ given by (4.2) and (4.3), respectively, can generate a pair of biorthogonal multiwavelets $\Psi(x)$ and $\tilde{\boldsymbol{\Psi}}(x)$ associated with $\boldsymbol{\Phi}(x)$ and $\tilde{\boldsymbol{\Phi}}(x)$.

Moreover, if $\Phi(x)$ and $\tilde{\Phi}(x)$ are continuous and interpolatory, then $\Psi(x)$ and $\tilde{\Psi}(x)$ have the same interpolatory property, that is,

$$
\boldsymbol{\Psi}(k)=\tilde{\Psi}(k)=\delta_{k, 0} \mathbf{e}_{1} \quad \text { and } \quad \Psi\left(k+\frac{1}{2}\right)=\tilde{\Psi}\left(k+\frac{1}{2}\right)=\delta_{k, 0} \mathbf{e}_{2}, \quad k \in Z .
$$

Proof. To prove Theorem 4.1, we need to check that the symbols $P(z), \tilde{P}(z)$, $Q(z)$ and $\tilde{Q}(z)$ satisfy (4.1). In fact,

$$
P(z) \tilde{Q}(z)^{*}=\frac{1}{4}\left[\begin{array}{cc}
1-H(z) \tilde{H}(z)^{*} & \bar{z}-\frac{1}{8} \bar{z}^{2 p+1} H(z) H(-z) \\
z-g z^{2 p+1} \tilde{H}(-z)^{*} \tilde{H}(z)^{*} & 1-H(z) \tilde{H}(z)^{*}
\end{array}\right] .
$$

Hence $P(z) \tilde{Q}(z)^{*}+P(-z) \tilde{Q}(-z)^{*}=O$. In the same way,

$$
\tilde{P}(z) Q(z)^{*}+\tilde{P}(-z) Q(-z)^{*}=O \quad \text { and } \quad Q(z) \tilde{Q}(z)^{*}+Q(-z) \tilde{Q}(-z)^{*}=I_{2} .
$$

Noticing that $Q(z)$ defined in (4.2) is the symbol of the sequence $\left\{Q_{k}\right\}$ associated with $\Psi(x)$ and applying (2.2), we have

$$
\boldsymbol{\Psi}(k)=\sum_{\ell \in Z} Q_{\ell} \boldsymbol{\Phi}(2 k-\ell)=Q_{2 k} \mathbf{e}_{1}=\delta_{k, 0} \mathbf{e}_{1}, \quad k \in Z .
$$

Similarly,

$$
\Psi\left(k+\frac{1}{2}\right)=\sum_{\ell \in Z} Q_{\ell} \Phi(2 k+1-\ell)=Q_{2 k+1} \mathbf{e}_{1}=\delta_{k, 0} \mathbf{e}_{2}, \quad k \in Z .
$$

In the same way, we can prove that $\boldsymbol{\Psi}(k)=\delta_{k, 0} \mathbf{e}_{1}$ and $\boldsymbol{\Psi}(k+1 / 2)=\delta_{k, 0} \mathbf{e}_{2}, k \in Z$. This means that $\Psi(x)$ and $\tilde{\Psi}(x)$ are interpolatory. This completes the proof.

\section{Improvement of the Corresponding Multiwavelet Frame}

The multiscaling function $\Phi(x)=\left(\phi_{1}, \phi_{2}\right)^{T}$ is said to be stable if there are two positive constants $0<A \leq B<\infty$ such that for any $\mathrm{f}(x) \in V_{N}$

$$
A\|\mathbf{f}\|^{2} \leq \sum_{i=1}^{2} \sum_{\ell \in Z}\left|\left\langle\mathbf{f}(x), \phi_{i: N, \ell}(x)\right\rangle\right|^{2} \leq B\|\mathbf{f}\|^{2} .
$$


A frame is called a tight frame, if $A=B$. Let $\Phi(x)$ and $\tilde{\Phi}(x)$ be a pair of biorthogonal multiscaling functions. Further, suppose $V_{N}=\widetilde{V}_{N}$. Then in terms of frame theory,

$$
\frac{1}{B}\|\mathbf{f}\|^{2} \leq \sum_{i=1}^{2} \sum_{\ell \in Z}\left|\left\langle\mathbf{f}(x), \tilde{\phi}_{i: N, \ell}(x)\right\rangle\right|^{2} \leq \frac{1}{A}\|\mathbf{f}\|^{2}
$$

and

$$
\|\mathbf{f}\|^{2}=\langle\mathbf{f}, \mathbf{f}\rangle=\sum_{i=1}^{2} \sum_{\ell \in Z}\left\langle\mathbf{f}(x), \phi_{i: N, \ell}(x)\right\rangle\left\langle\mathbf{f}(x), \tilde{\phi}_{i: N, \ell}(x)\right\rangle .
$$

By Theorem 3.3, we have, $\forall 0<\alpha<1$,

$$
\mathbf{f}(x)=\sum_{\ell \in Z} 2^{-N / 2}\left[f\left(\frac{\ell}{2^{N}}\right), f\left(\frac{\ell}{2^{N}}+\frac{1}{2^{N+1}}\right)\right]\left[\alpha \Phi_{N, \ell}(x)+(1-\alpha) \tilde{\Phi}_{N, \ell}(x)\right] .
$$

Equation (5.3) means that $\left\{\alpha \Phi_{N, \ell}(x)+(1-\alpha) \tilde{\Phi}_{N, \ell}(x)\right\}$ constitutes a frame.

As we know, the more approximate a frame bounding $A$ and $B$ is, the stabler the frame is. In terms of frame theory, we found an interesting result, as follows.

THEOREM 5.1. Under the conditions of Theorem 3.3, suppose that $\boldsymbol{\Phi}(x)$ satisfies (5.1), and $V_{N}=\widetilde{V}_{N}$. Then $\left\{\alpha \boldsymbol{\Phi}_{N, \ell}(x)+(1-\alpha) \tilde{\Phi}_{N, \ell}(x)\right\}$, where $0<\alpha<1$, also form a frame, and one which is stabler than the frame $\left\{\Phi_{N, \ell}(x)\right\}$.

PROOF. Let $\beta=1-\alpha$. Thus, we have

$$
\begin{aligned}
\sum_{i=1}^{2} \sum_{\ell \in Z}\left|\left\langle\mathbf{f}(x), \alpha \phi_{i: N, \ell}(x)+(1-\alpha) \tilde{\phi}_{i: N, \ell}(x)\right\rangle\right|^{2} \\
=\sum_{i=1}^{2} \sum_{\ell \in Z}\left|\left\langle\mathbf{f}(x), \alpha \phi_{i: N, \ell}(x)+\beta \tilde{\phi}_{i: N, \ell}(x)\right\rangle\right|^{2} \\
\left.=\sum_{i=1}^{2} \sum_{\ell \in Z} \alpha^{2}\left|\left\langle\mathbf{f}(x), \phi_{i: N, \ell}(x)\right\rangle\right|^{2}+\beta^{2}|| \mathbf{f}(x), \tilde{\phi}_{i: N, \ell}(x)\right\rangle\left.\right|^{2} \\
\quad+2 \alpha \beta\left\langle\mathbf{f}(x), \phi_{i: N, \ell}(x)\right\rangle\left\langle\mathbf{f}(x), \tilde{\phi}_{i: N, \ell}(x)\right\rangle \\
\left.=\sum_{i=1}^{2} \sum_{\ell \in Z} \alpha^{2}|| \mathbf{f}(x), \phi_{i: N, \ell}(x)\right\rangle\left.\right|^{2}+\beta^{2}\left|\left\langle\mathbf{f}(x), \tilde{\phi}_{i: N, \ell}(x)\right\rangle\right|^{2}+2 \alpha \beta\|\mathbf{f}\|^{2} .
\end{aligned}
$$

In connection with (5.1) and (5.2), we have

$$
\begin{aligned}
\left(\alpha^{2} A+\beta^{2} \frac{1}{B}+2 \alpha \beta\right)\|\mathbf{f}\|^{2} & \left.\leq \sum_{i=1}^{2} \sum_{\ell \in Z} \| \mathbf{f}(x), \alpha \phi_{i: N, \ell}(x)+\beta \tilde{\phi}_{i: N, \ell}(x)\right)\left.\right|^{2} \\
& \leq\left(\alpha^{2} B+\beta^{2} \frac{1}{A}+2 \alpha \beta\right)\|\mathbf{f}\|^{2}
\end{aligned}
$$


The above inequalities mean that $\left\{\alpha \Phi_{N, \ell}(x)+(1-\alpha) \tilde{\boldsymbol{\Phi}}_{N, \ell}(x)\right\}$ is a frame, and with frame bounds $\alpha^{2} A+\beta^{2} / B+2 \alpha \beta$ and $\alpha^{2} B+\beta^{2} / A+2 \alpha \beta$. Since $A<B$,

$$
\alpha^{2} A B+2 \alpha \beta A+\beta^{2}<\alpha^{2} A B+2 \alpha \beta B+\beta^{2} .
$$

That is, $A\left[\alpha^{2} B+2 \alpha \beta+\beta^{2} / A\right]<B\left[\alpha^{2} A+2 \alpha \beta+\beta^{2} / B\right]$. Hence we have the following inequality:

$$
\frac{A}{B}<\frac{\alpha^{2} A+\beta^{2} \frac{1}{B}+2 \alpha \beta}{\alpha^{2} B+\beta^{2} \frac{1}{A}+2 \alpha \beta} .
$$

The above inequalities imply that the frame $\left\{\alpha \boldsymbol{\Phi}_{N, \ell}(x)+(1-\alpha) \tilde{\boldsymbol{\Phi}}_{N, \ell}(x)\right\}$ is stabler than the frame $\left\{\Phi_{N, \ell}(x)\right\}$.

\section{Example}

We will provide an illustrative example of how to use our method to construct biorthogonal interpolatory multiscaling functions and multiwavelets.

EXAMPLE 1. Let

$$
\begin{aligned}
& H(z)=-\frac{\sqrt{2}}{8} z^{-2}+\frac{\sqrt{2}}{4} z^{-1}+\frac{3 \sqrt{2}}{4}+\frac{\sqrt{2}}{4} z-\frac{\sqrt{2}}{8} z^{2} \text { and } \\
& \tilde{H}(z)=\frac{\sqrt{2}}{4} z^{-1}+\frac{\sqrt{2}}{2}+\frac{\sqrt{2}}{4} z .
\end{aligned}
$$

One can check that $H(z)$ and $\tilde{H}(z)$ satisfy (3.7). Suppose $g=1$ and $p=0$. By (3.11),

$$
\begin{aligned}
& G(z)=-\frac{\sqrt{2}}{4} z^{2}+\frac{\sqrt{2}}{2} z-\frac{\sqrt{2}}{4} \text { and } \\
& \tilde{G}(z)=-\frac{\sqrt{2}}{8} z^{3}-\frac{\sqrt{2}}{4} z^{2}+\frac{3 \sqrt{2}}{4} z-\frac{\sqrt{2}}{4}-\frac{\sqrt{2}}{8} z^{-1} .
\end{aligned}
$$

Using (3.5), we obtain

$$
\begin{aligned}
& P(z)=\frac{1}{2}\left[\begin{array}{cc}
1 & -\frac{\sqrt{2}}{8} z^{-2}+\frac{\sqrt{2}}{4} z^{-1}+\frac{3 \sqrt{2}}{4}+\frac{\sqrt{2}}{4} z-\frac{\sqrt{2}}{8} z^{2} \\
z & -\frac{\sqrt{2}}{4} z^{2}+\frac{\sqrt{2}}{2} z-\frac{\sqrt{2}}{4}
\end{array}\right] \text { and } \\
& \tilde{P}(z)=\frac{1}{2}\left[\begin{array}{cc}
1 & \frac{\sqrt{2}}{4} z^{-1}+\frac{\sqrt{2}}{2}+\frac{\sqrt{2}}{4} z \\
z & -\frac{\sqrt{2}}{8} z^{3}-\frac{\sqrt{2}}{4} z^{2}+\frac{3 \sqrt{2}}{4} z-\frac{\sqrt{2}}{4}-\frac{\sqrt{2}}{8} z^{-1}
\end{array}\right] .
\end{aligned}
$$

Applying Theorem 3.2, $P(z)$ and $\widetilde{P}(z)$ defined in (6.1) and (6.2) respectively, can generate a pair of biorthogonal interpolatory multiscaling functions $\boldsymbol{\Phi}(x)$ and $\tilde{\boldsymbol{\Phi}}(x)$. 
By (4.2) and (4.3), we have

$$
\begin{array}{rlrl}
Q_{-2} & =\left[\begin{array}{cc}
0 & -\frac{\sqrt{2}}{8} \\
0 & 0
\end{array}\right], & Q_{-1}=\left[\begin{array}{cc}
0 & -\frac{\sqrt{2}}{4} \\
0 & 0
\end{array}\right], & Q_{0}=\left[\begin{array}{cc}
1 & -\frac{3 \sqrt{2}}{4} \\
0 & \frac{1}{4}
\end{array}\right], \\
Q_{1}=\left[\begin{array}{cc}
0 & -\frac{\sqrt{2}}{4} \\
1 & -\frac{\sqrt{2}}{2}
\end{array}\right], & Q_{2}=\left[\begin{array}{ll}
0 & \frac{\sqrt{2}}{8} \\
0 & \frac{\sqrt{2}}{4}
\end{array}\right], & \text { and } \\
\tilde{Q}_{-1}=\left[\begin{array}{cc}
0 & -\frac{\sqrt{2}}{4} \\
0 & \frac{\sqrt{2}}{8}
\end{array}\right], & \tilde{Q}_{0}=\left[\begin{array}{cc}
1 & -\frac{\sqrt{2}}{2} \\
0 & \frac{\sqrt{2}}{4}
\end{array}\right], & \tilde{Q}_{1}=\left[\begin{array}{cc}
0 & -\frac{\sqrt{2}}{4} \\
1 & -\frac{3 \sqrt{2}}{4}
\end{array}\right], \\
\tilde{Q}_{2}=\left[\begin{array}{cc}
0 & 0 \\
0 & \frac{\sqrt{2}}{4}
\end{array}\right] & \text { and } & \tilde{Q}_{3}=\left[\begin{array}{ll}
0 & 0 \\
0 & \frac{\sqrt{2}}{8}
\end{array}\right] . &
\end{array}
$$

By Theorem 4.1, $Q(z)=(1 / 2) \sum_{k=-2}^{2} Q_{k} z^{k}$ and $\tilde{Q}(z)=(1 / 2) \sum_{k=-1}^{3} \tilde{Q}_{k} z^{k}$ can generate a pair of biorthogonal interpolatory multiwavelets $\Psi(x)$ and $\tilde{\Psi}(x)$ associated with $\boldsymbol{\Phi}(x)$ and $\tilde{\boldsymbol{\Phi}}(x)$.

\section{Acknowledgements}

The author wishes to thank the referees for their valuable suggestions and comments which have improved the presentation of the results.

This research was supported by the Natural Science Foundation of Guangdong Province (Grants 06105648, 05008289 and 032038), and the Doctoral Foundation of Guangdong Province (Grant 04300917).

\section{References}

[1] C. K. Chui and J. Lian, "A study on orthonormal multiwavelets", J. Appl. Numer. Math. 20 (1996) 273-298.

[2] C. K. Chui and J. Z. Wang, "A cardinal spline approach to wavelets", Proc. Amer. Math. Soc. 113 (1991) 785-793.

[3] I. Daubechies and J. C. Lagarias, "Two-scale difference equations. II. Local regularity, infinite products of matrices and fractals", SIAM J. Math. Anal. 23 (1991) 1031-1079.

[4] G. C. Donovan, J. S. Geronimo and D. P. Hardin, "Construction of orthogonal wavelets using fractal interpolation functions", SIAM J. Math. Anal. 27 (1996) 1158-1192.

[5] J. S. Geronimo, D. P. Hardin and P. R. Massopust, "Fractal functions and wavelet expansions based on several scaling functions", J. Approx. Theory 78 (1994) 371-401.

[6] S. S. Goh, Jiang Qingtang and Xia Tao, "Construction of biorthogonal multiwavelets using the lifting scheme", Appl. Comput. Harmon. Anal. 9 (2000) 336-352.

[7] T. N. T. Goodman, S. L. Lee and W. S. Tang, "Wavelets in wandering subspaces", Trans. Amer. Math. Soc. 338 (1993) 639-654. 
[13] Biorthogonal interpolatory multiscaling functions and corresponding multiwavelets

[8] J. Lian, "Orthogonal criteria for multiscaling functions", Appl. Comp. Harm. Anal. 5 (1998) $277-311$.

[9] G. C. Rota and G. Strang, “A note on the joint spectral radius", Indag. Math. 22 (1960) 379-381.

[10] Yang Shouzhi, "A fast algorithm for constructing orthogonal multiwavelets", ANZIAM J. 46 (2004) 185-202.

[11] Yang Shouzhi, "An algorithm for constructing biorthogonal multiwavelets with higher approximation orders", ANZIAM J. 47 (2006) 513-526.

[12] Yang Shouzhi, Cheng Zhengxing and Wang Hongyong, "Construction of biorthogonal multiwavelets", J. Math. Anal. Appl. 276 (2002) 1-12.

[13] G. Strang and D. X. Zhou, "Inhomogeneous refinement equations", J. Fourier Anal. Appl. 4 (1998) 733-747.

[14] X. G. Xia and Z. Zhang, "On sampling theorem, wavelets, and wavelet transforms", IEEE Trans. Signal Processing 41 (1993) 3524-3535.

[15] D. X. Zhou, "Existence of multiple refinable distributions", Michigan Math. J. 44 (1997) 317-329.

[16] D. X. Zhou, "The p-norm joint spectral radius for even integers", Methods Appl. Anal. 5 (1998) 39-54.

[17] D. X. Zhou, "Multiple refinable Hermite interpolants", J.Approx. Theory 102 (2000) 46-71.

[18] D. X. Zhou, "Interpolatory orthogonal multiwavelets and refinable functions", IEEE Trans. Signal Processing 50 (2002) 520-527. 